\title{
Magnetic field and dynamic pressure ULF fluctuations in coronal-mass-ejection-driven sheath regions
}

\author{
E. K. J. Kilpua ${ }^{1}$, H. Hietala ${ }^{2}$, H. E. J. Koskinen ${ }^{1,3}$, D. Fontaine ${ }^{4}$, and L. Turc ${ }^{4}$ \\ ${ }^{1}$ Department of Physics, P.O. Box 64, University of Helsinki, Finland \\ ${ }^{2}$ Space and Atmospheric Physics Group, The Blackett Laboratory, Imperial College, London SW7 2AZ, UK \\ ${ }^{3}$ Finnish Meteorological Institute, P.O. Box 503, Helsinki, Finland \\ ${ }^{4}$ Laboratoire de Physique des Plasmas, Ecole Polytechnique, Route de Saclay 91128 Palaiseau, France
}

Correspondence to: E. K. J. Kilpua (emilia.kilpua@ helsinki.fi)

Received: 3 March 2013 - Revised: 27 May 2013 - Accepted: 2 August 2013 - Published: 10 September 2013

\begin{abstract}
Compressed sheath regions form ahead of interplanetary coronal mass ejections (ICMEs) that are sufficiently faster than the preceding solar wind. The turbulent sheath regions are important drivers of magnetospheric activity, but due to their complex internal structure, relatively little is known on the distribution of the magnetic field and plasma variations in them. In this paper we investigate ultra low frequency (ULF) fluctuations in the interplanetary magnetic field (IMF) and in dynamic pressure $\left(P_{\text {dyn }}\right)$ using a superposed epoch analysis of 41 sheath regions observed during solar cycle 23 . We find strongest fluctuation power near the shock and in the vicinity of the ICME leading edge. The IMF and $P_{\text {dyn }}$ ULF power have different profiles within the sheath; the former is enhanced in the leading part of the sheath, while the latter is increased in the trailing part of the sheath. We also find that the ICME properties affect the level and distribution of the ULF power in sheath regions. For example, sheath regions associated with strong or fast ICMEs, or those that are crossed at intermediate distances from the center, have strongest ULF power and large variation in the power throughout the sheath region. The weaker or slower ICMEs, or those that are crossed centrally, have in general considerably weaker ULF power with relatively smooth profiles. The strong and abrupt decrease of the IMF ULF power at the ICME leading edge could be used to distinguish the ICME from the preceding sheath plasma.
\end{abstract}

Keywords. Interplanetary physics (interplanetary magnetic fields; solar wind plasma) - Space plasma physics (waves and instabilities)

\section{Introduction}

Coronal mass ejections (CMEs) are spectacular eruptions of solar plasma and magnetic field into the interplanetary space. When an interplanetary CME (ICME) moves supermagnetosonically with respect to the ambient solar wind, a fast forward shock develops ahead of it. The solar wind plasma and interplanetary magnetic field (IMF) are compressed at the shock and pile up in the front of the ICME to form a turbulent sheath region. It is now well established that ICME sheath regions cause intense magnetospheric storms (Tsurutani et al., 1988; Huttunen et al., 2002). The effect of sheath regions is particularly strong in the high-latitude magnetosphere (Huttunen and Koskinen, 2004). There the rapid variations in the ionospheric current systems are associated with auroral displays and space weather hazards, such as geomagnetically induced currents in the pipelines and electric transfer systems (Huttunen et al., 2008).

It is likely that the turbulent structure of sheath regions contributes to their distinct geomagnetic effects. There is increasing evidence that fluctuations in the interplanetary magnetic field (IMF) and in solar wind dynamic pressure $\left(P_{\text {dyn }}\right)$ can have a substantial contribution to how solar wind energy transfers to the magnetosphere-ionosphere system and the global ionospheric and magnetospheric convection pattern. Kim et al. (2009) showed that the IMF north-south component $\left(B_{Z}\right)$ and $P_{\text {dyn }}$ fluctuations in the ultra low frequency (ULF) range can significantly influence the dayside ionospheric convection. The solar wind ULF fluctuations have a clear effect also on the nightside plasma sheet dynamics and 
structure as well as on the substorm occurrence (Lyons et al., 2009). In addition, Borovsky and Funsten (2002) demonstrated that the enhanced upstream IMF turbulence increases the momentum transfer from the magnetosheath into the magnetosphere, resulting in more stirring of the magnetosphere and higher geomagnetic activity.

Previous studies have also shown that the upstream solar wind ULF fluctuations in $P_{\text {dyn }}$ and IMF from a few minutes to $10 \mathrm{~min}$ have a strong correlation with similar frequency range fluctuations in the magnetosphere (e.g., Kepko et al., 2005; Kessel et al., 2004; Kessel, 2010; Simms et al., 2008). In the magnetospheric context these waves are called Pc5 fluctuations. These fluctuations and their correlation with the upstream solar wind conditions have been studied intensively due to the importance of Pc5 waves in the energetic particle transport and energization of electrons to relativistic levels (e.g., Baker et al., 1998).

The internal structure of sheath regions is highly complex as they are composed of different layers of IMF and solar wind plasma that accumulate over the several days it takes for a CME to travel from the Sun to the Earth. The downstream region close to the ICME-driven shock is known to have large amplitude magnetic field variations (e.g., Kataoka et al., 2005). However, relatively little is known on how the magnetic field and solar wind plasma fluctuations are distributed within the sheath, and how they depend on the driver and shock properties.

The variations of the small-scale turbulence in the Earth's magnetosheath from the bow shock to the magnetopause have been studied relatively extensively (e.g., Luhmann et al., 1986; Schwartz et al., 1996; Sahraoui et al., 2003; Lucek et al., 2005; Shevyrev et al., 2005; Yordanova et al., 2008), but there are distinct differences between the ICME sheaths and the planetary magnetosheaths (Kaymaz and Siscoe, 2006; Siscoe and Odstrcil, 2008). As pointed out by (Kaymaz and Siscoe, 2006), the differences are likely attributed to the long history of accretion of inhomogenous IMF and plasma in ICME sheath regions. In addition, ICME sheath regions are a combination of a "propagation sheath" and an "expansion sheath" (Siscoe and Odstrcil, 2008): the former refers to the sheath that forms around an object propagating relative to solar wind, and the latter is due to an expanding object.

In this paper we investigate the level and distribution of IMF and $P_{\text {dyn }}$ ULF waves in ICME sheath regions using a superposed epoch analysis. We concentrate on the interval from 3 to $10 \mathrm{~min}$, which approximately corresponds to the magnetospheric Pc5 range. We also group the events according to the driver ICME and shock characteristics. The paper is organized as follows. In Sect. 2 we present the methods and definitions used. In Sect. 3 we present our statistical results, and in Sects. 4 and 5 we discuss and summarize our findings.

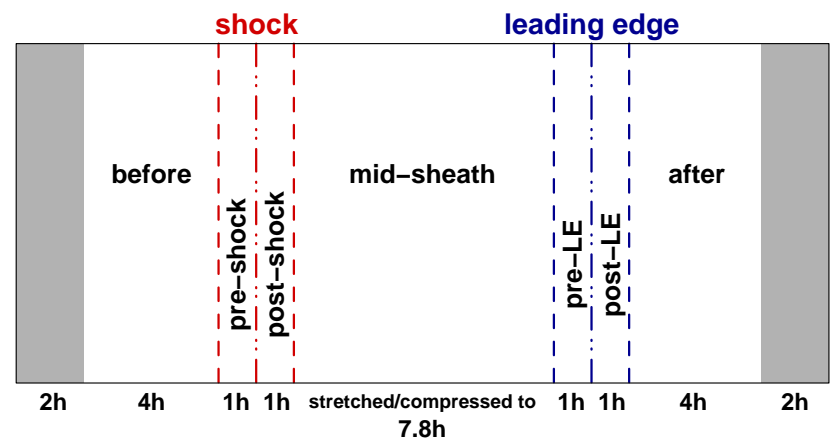

Fig. 1. The panel shows different regions and their durations used in this analysis. The red dashed line marks the shock, and the blue dashed line indicates the ICME leading edge. The gray domains in the beginning and the end of the displayed region are the intervals that are included in the calculation of the wavelet spectrograms, but not included in the actual analysis.

\section{Data and methods}

We use ACE $16 \mathrm{~s}$ level 2 magnetic field data from MAG (Smith et al., 2008) and $64 \mathrm{~s}$ level 2 plasma data from SWE (Ogilvie et al., 1995). The data are obtained through CDAWeb. ACE is positioned at the Lagrangian point L1, roughly 1.5 million kilometers upstream from the Earth.

The sheath events are selected using the ACE ICME cata$\log$ maintained at the ACE Science Center. In the ACE catalog, ICMEs are divided into three groups according to the spacecraft closest approach distance from the ICME center (i.e., the impact parameter). This categorization (Jian et al., 2006) is based on the time variations of the total pressure (sum of the magnetic pressure and plasma thermal pressure) perpendicular to the magnetic field $(P t)$ within the ICME: in Group 1 the $P t$ profile has a central maximum, in Group 2 it has a plateau-like profile, and in Group 3 it has a gradual decrease after a sharp increase at the ICME leading edge. ICMEs in Group 1 are crossed close to the center, while for Group 2 and Group 3 the impact parameter from the center increases (see the summary from Table 1). It is often difficult to determine the leading edge time for Group 3 ICMEs (many Group 3 ICMEs in the ACE catalog lack the leading edge time), and therefore we include here only Group 1 and Group 2 events.

In total, our data set comprises 41 sheath regions observed during solar cycle 23 (1998-2006). The average duration of these sheath regions is $9.8 \mathrm{~h}$. The durations range from 3.1 to $28.1 \mathrm{~h}$ with a standard deviation of $4.9 \mathrm{~h}$.

We calculate the Morlet wavelet spectra from seven hours before the shock to seven hours after the ICME leading edge. The cone of influence, determined by the wavelet analysis, is a region where the edge effects due to the use of finitelength series become important. In this study we restrict to data outside the cone of influence. When we calculate the 
Table 1. $P t$ categorization according to Jian et al., 2006.

\begin{tabular}{llll}
\hline & Group 1 & Group 2 & Group 3 \\
\hline $\begin{array}{l}\text { Pt profile } \\
\text { Impact parameter }\end{array}$ & central maximum & plateau & sharp increase, gradual decrease \\
glancing encounter
\end{tabular}

Table 2. Division of events with different criteria. The first column gives the name shown in Fig. 4, the second column the definition, and the last column the number of events with that criteria.

\begin{tabular}{lll}
\hline Label in Fig. 4 & Definition & Number of events \\
\hline Group 1 & central encounter & 19 \\
Group 2 & intermediate distance & 22 \\
\hline Fast ICME & ICME maximum speed $>550 \mathrm{~km} \mathrm{~s}^{-1}$ & 18 \\
Slow ICME & ICME maximum speed $<550 \mathrm{~km} \mathrm{~s}^{-1}$ & 23 \\
\hline Strong ICME & ICME leading edge magnetic field $>15 \mathrm{nT}$ & 19 \\
Weak ICME & ICME leading edge magnetic field $<15 \mathrm{nT}$ & 22 \\
\hline Strong shock & downstream-to-upstream magnetic field strength ratio $>2.0$ & 17 \\
Weak shock & downstream-to-upstream magnetic field strength ratio $<2.0$ & 24 \\
\hline
\end{tabular}

ULF power spectra we exclude two hours from both ends of the initial interval.

To investigate the ULF fluctuation power within and close to the sheath regions we define seven domains that are summarized schematically in Fig. 1. The regions are as follows: before, pre-shock, post-shock, mid-sheath, pre-LE, post-LE, and after, where LE stands for the (ICME) "leading edge". The before and after regions have duration of four hours and the pre-shock, post-shock, pre-LE, and post-LE regions last one hour.

The duration of the mid-sheath region varies from event to event. For the superposed epoch analysis we stretch or compress all mid-sheath regions to $7.8 \mathrm{~h}$ corresponding to the average sheath duration of $9.8 \mathrm{~h}$ where the $1 \mathrm{~h}$ post-shock and pre-LE regions have been subtracted. Note that the stretching of ULF power has been done after the wavelet analysis.

We separate the aforementioned $1 \mathrm{~h}$ regions around the shock and the leading edge because these are the regions where important microscale physical processes are expected to take place (e.g., Schwartz et al., 1996; Lucek et al., 2005; Blanco-Cano et al., 2006; Yordanova et al., 2008; Desai et al., 2011). We emphasize that these regions are not stretched/compressed or otherwise modified for the superposed epoch analysis.

To examine how the ICME and shock properties affect the distribution and level of ULF fluctuation in the sheath, we divide our events with the following criteria (see Table 2) to (1) Group 1 and Group 2 events, (2) slow and fast ICMEs using the ICME leading edge speed, (3) strong and weak ICMEs using the ICME leading edge magnetic field, and (4) strong and weak shocks using the downstream-toupstream magnetic field strength ratio. Our choice to use magnetic field strength ratio instead of Mach number to estimate the shock strength is based on large uncertainties that would be introduced when calculating the Mach number from single spacecraft measurements with an algorithmic approach for our statistical study. Further, magnetic field measurements are more accurate/reliable than plasma density measurements.

Figure 2 shows an example event that occurred on 14 December 2006. The shock was observed at ACE on 14 December at 13:52 UTC and the ICME leading edge on 14 December, 22:30 UTC. For this ICME the leading edge magnetic field was $18.6 \mathrm{nT}$, and the leading edge speed $890 \mathrm{~km} \mathrm{~s}^{-1}$. According to the ACE ICME catalog this ICME was crossed at an intermediate distance from the center (Group 2). The duration of the mid-sheath region for the event shown in Fig. 2 is 5.6 h.

The wavelet spectrograms for the GSM $B_{Z}$ and $P_{\text {dyn }}$ are shown in Fig. 2b and e, respectively. We use here GSM components because it is a natural coordinate system when investigating the solar wind interaction with the magnetosphere. The pair of horizontal lines bounds the ULF range, and the fluctuation powers calculated in that range are shown in Fig. 2c and f, respectively. For the superposed epoch analysis we calculate the wavelet spectrograms and the fluctuation power for $B_{X}$ and $B_{Y}$ in the similar manner as for $B_{Z}$. Figure 2c shows that the $B_{Z}$ power was high in the postshock region and during the first part of the mid-sheath, but then decreased considerably. The power increased again in 


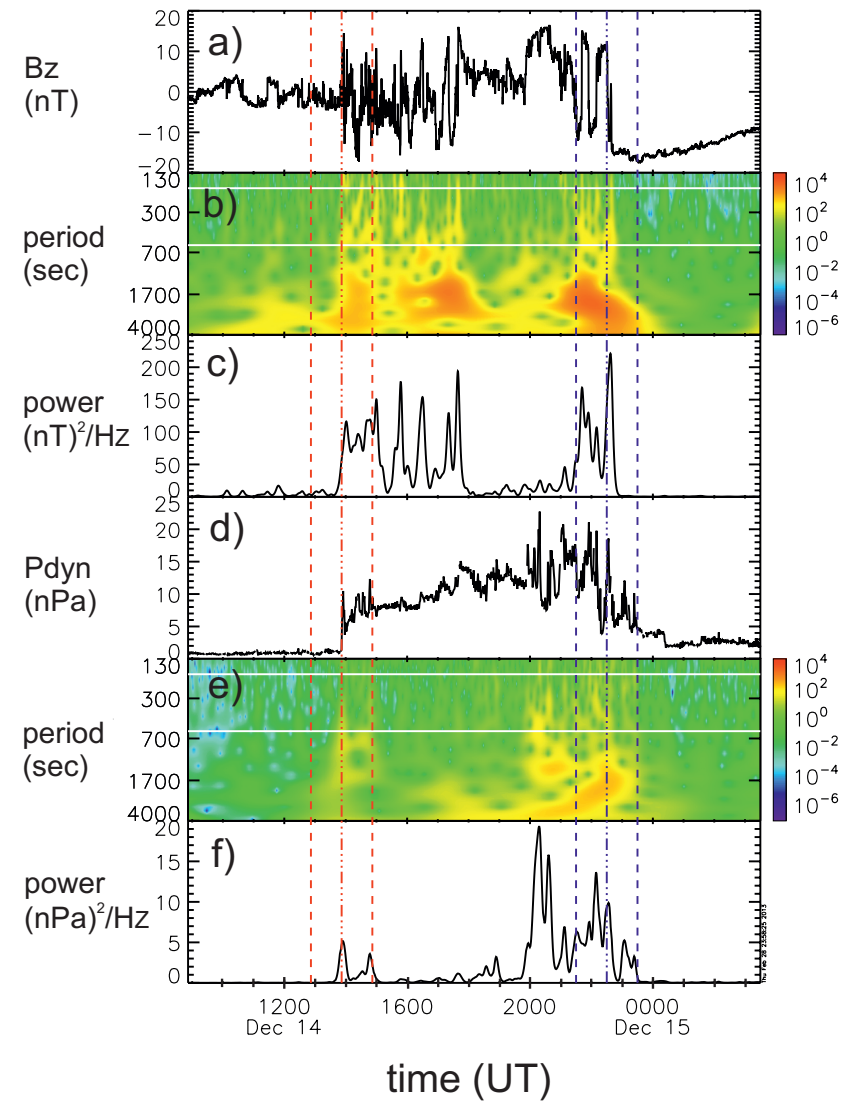

Fig. 2. Example event on 14 December 2006. The panels show the following from top to bottom: (a) IMF $Z$ component in GSM $\left(B_{Z}\right)$, (b) wavelet spectrogram for $B_{Z}$, (c) $B_{Z}$ ULF power, (d) solar wind $P_{\text {dyn }}$, (e) wavelet spectrogram for $P_{\text {dyn }}$, and (f) $P_{\text {dyn }}$ ULF power. The red dash-dotted line marks the shock, and the blue dash-dotted line the leading edge. The $1 \mathrm{~h}$ regions adjacent to the shock and the leading edge are bounded by the pairs of dashed lines. In panels (b) and (e) the pair of white lines bounds the 3 to 10 min frequency range we have used to calculate the ULF powers in panels (c) and (f).

the pre-LE region, after which it decreased sharply at the leading edge and remained low in the post-LE region and during the first hours of the ICME. In contrast, the $P_{\mathrm{dyn}}$ ULF power (Fig. 2f) was highest during the latter part of the midsheath and in the pre-LE region. Weaker enhancements were observed in the post-shock and post-LE regions.

\section{Statistical results}

\subsection{Superposed epoch analysis}

Figure 3 shows the results of the superposed epoch analysis for the IMF GSM components and $P_{\text {dyn }}$. We see that the variations in $B_{Z}$ and $B_{Y}$ are roughly similar in different regions. The largest power in the fluctuations is in $B_{Z}$, while the lowest fluctuation power is evidently in $B_{X}$. In the following, we

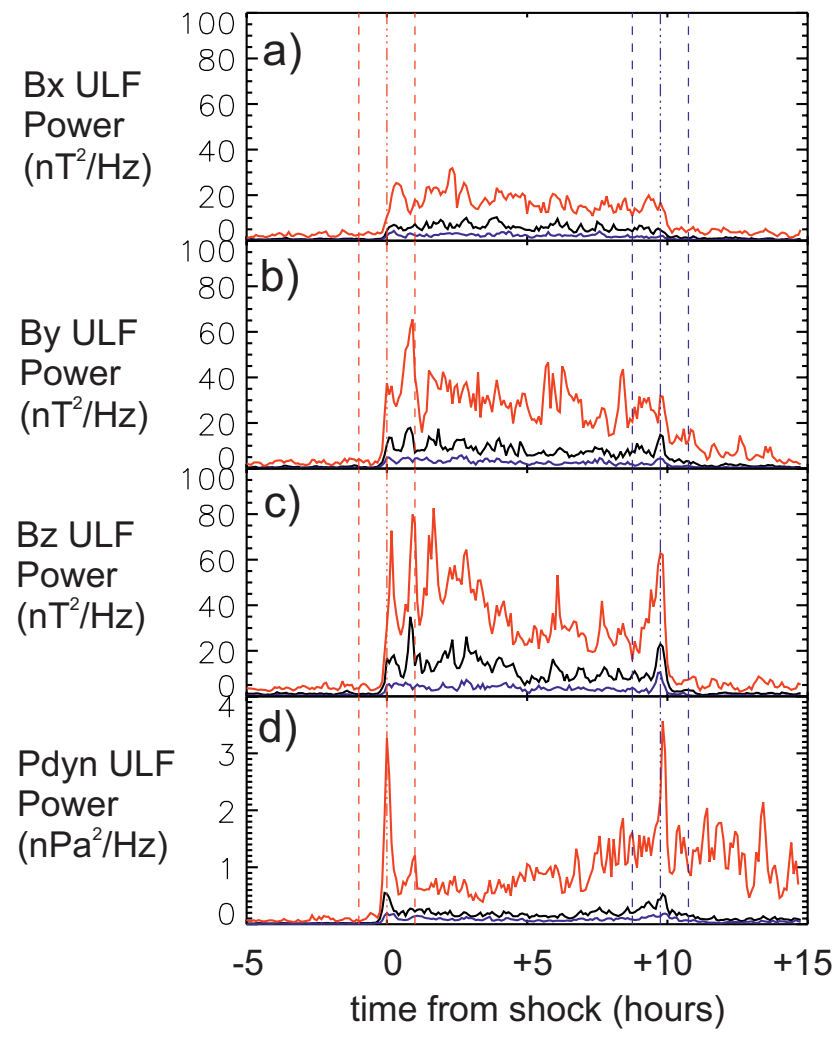

Fig. 3. The results of the superposed epoch analysis of the 41 studied events. The curves show the median (black), and the upper (red) and lower (blue) quartiles for ULF power in (a) $B_{X}$, (b) $B_{Y}$, (c) $B_{Z}$, and (d) $P_{\text {dyn }}$ fluctuations. The red dash-dotted line shows the shock, and the blue dash-dotted line the leading edge. The $1 \mathrm{~h}$ (non-stretched) regions adjacent to the shock and the leading edge are bounded by the pairs of dashed lines.

will use $B_{Z}$ as the representative IMF component, but we will note if there were clear differences in the characteristics between different components. In Table 3 we also give quartile powers of $B_{Z}$ and $P_{\mathrm{dyn}}$ fluctuations in different regions. Due to the behavior of $B_{Z}$ and $P_{\mathrm{dyn}}$ ULF powers described below, we have separated the mid-sheath region into the leading and trailing parts in Table 3.

We immediately notice that the $B_{Z}$ ULF power is significantly higher throughout the sheath region when compared to the surrounding solar wind. The transition out of the sheath region is marked at both edges by strong and abrupt decrease in the fluctuation power. From Table 3 we also see that the $B_{Z}$ power in the before, pre-shock, and after regions is lower than in the other regions. Figure $3 \mathrm{a}$ and Table 3 show that the $B_{Z}$ ULF power peaks in the post-shock and pre-LE regions. For the lower quartile power the strongest peak is in the pre-LE region. Note that for $B_{Y}$ there is no obvious peak for the upper quartile power in the pre-LE region, but it peaks strongly in the post-shock region. The $B_{Z}$ power is obviously higher in the first half of the mid-sheath than in the second half: from the values shown in Table 3 we can 
Table 3. The averages of the lower quartile, median, and upper quartile $B_{Z}$ and $P_{\text {dyn }}$ ULF powers in different regions (see Fig. 1). Note that we have separated here the mid-sheath into the leading and trailing sections. The units are $\left(\mathrm{nT}^{2} \mathrm{~Hz}^{-1}\right)$ and $\left(\mathrm{nPa}^{2} \mathrm{~Hz}^{-1}\right)$ for the $B Z$ and $P_{\text {dyn }}$ ULF powers, respectively.

\begin{tabular}{llll|lll}
\hline Region & $B_{Z}$ lower & $B_{Z}$ median & $B_{Z}$ upper & $P_{\text {dyn }}$ lower & $P_{\text {dyn }}$ median & $P_{\text {dyn }}$ upper \\
\hline Before & 0.43 & 1.21 & 3.26 & 0.0067 & 0.016 & 0.049 \\
Pre-shock & 0.46 & 1.65 & 4.63 & 0.026 & 0.092 & 0.25 \\
Post-shock & 4.85 & 17.3 & 41.8 & 0.11 & 0.28 & 0.96 \\
Leading mid-sheath & 4.18 & 14.8 & 40.5 & 0.084 & 0.17 & 0.48 \\
Trailing mid-sheath & 3.06 & 8.93 & 24.9 & 0.066 & 0.21 & 0.70 \\
Pre-LE & 3.92 & 11.3 & 33.2 & 0.11 & 0.32 & 1.01 \\
Post-LE & 1.35 & 5.58 & 12.6 & 0.092 & 0.25 & 1.15 \\
After & 0.33 & 1.24 & 4.33 & 0.036 & 0.092 & 0.55 \\
\hline
\end{tabular}

calculate that the power in the leading mid-sheath is 20,45 , and $68 \%$ higher than in the trailing mid-sheath for the lower quartile, median, and upper quartile powers, respectively. It is also seen from Fig. 3 that all three power curves for $B_{X}$ show considerably flatter profiles than for $B_{Y}$ and $B_{Z}$.

The $P_{\text {dyn }}$ ULF power (Fig. 3b) is also strongly elevated in the sheath region when compared with the preceding solar wind. For the median and lower quartile curves (see also Table 3 ) the power decreases after the leading edge, but for the strongest events (red upper quartile curve), the power levels stay high throughout the front part of the ICME. Similar to $B_{Z}$ ULF power, the $P_{\text {dyn }}$ ULF power peaks in the post-shock and pre-LE regions, but in contrast, the trailing half of the mid-sheath has stronger $P_{\text {dyn }}$ ULF power than the leading half. While the lower quartile and median power are only slightly higher in the trailing part (Table 3), for the strongest events the power in the trailing part is $117 \%$ higher than in the leading part. It is also seen from Table 3 that the lower quartile curve has a very flat distribution throughout the sheath. The large difference between the power levels of the upper quartile and median curves indicate that there were a few events with particularly strong $P_{\text {dyn }}$ ULF fluctuation power.

\subsection{Effect of ICME/shock properties on sheath ULF fluctuations}

We calculated the median and quartile curves of $B_{Z}$ and $P_{\text {dyn }}$ ULF power in each of the seven regions (Fig. 1) for different categories defined in Table 2. The results are shown in Fig. 4.

First, let us compare the level of power in the different categories (Table 2). We see that fast or strong ICMEs are associated with much higher ULF power both for $P_{\text {dyn }}$ and $B_{Z}$ than slow or weak ICMEs. The power is also somewhat higher in sheath regions of ICMEs that are crossed from intermediate distances from the center (Group 2) than centrally encountered ICMEs (Group 1). Sheath regions behind strong shocks have also stronger ULF power, in particular for $P_{\text {dyn }}$.
Next, we find that the distribution of ULF power between different regions has also distinct differences depending on the shock/driver properties. For Group 2 events the $B_{Z}$ power peaks in the post-shock region and in the first half of the mid-sheath, while for Group 1 events the $B_{Z}$ power clearly has a flatter profile. The main $B_{Z}$ power peak for Group 1 events occurs at the leading edge. The $P_{\text {dyn }}$ power has a sharp peak in the post-shock region, and is strongly enhanced in the latter part of the mid-sheath and in the pre-LE region for Group 2 events, while for Group 1 events a sharp peak is found in the post-LE region.

For fast ICMEs there is a very strong increase in the $B_{Z}$ power in the post-shock region and during the first part of the mid-sheath, while for slower ICMEs the increase in power close to the shock is more modest. $P_{\text {dyn }}$ ULF power shows a broad enhancement starting from the latter part of the midsheath for fast ICMEs, while for slow ICMEs the levels stay relatively smooth, except the upper quartile curve showing a sharp peak at the leading edge. The power peak in the postshock region is also considerably stronger for fast ICMEs than for slow ICMEs.

The distribution of $B_{Z}$ power seems rather similar in sheath regions behind strong and weak shocks. The only remarkable difference is that the peaks in the $B_{Z}$ power at sheath boundaries are more pronounced in the case of a strong shock. The $P_{\text {dyn }}$ power has the sharpest peak in the post-shock region for events associated with strong shocks, while for events with weak shocks the main enhancement is in the post-LE region. The $P_{\text {dyn }}$ power has also much larger variations in the mid-sheath region for the strong shock category than for the events associated with weak shocks.

The sheath regions of strong ICMEs exhibit high $B_{Z}$ power in the post-shock and pre-LE regions and during the first part of the mid-sheath. In turn, the sheath regions preceding weaker ICMEs have rather flat distribution, except for a peak in the first part of the mid-sheath. The $P_{\text {dyn }}$ power shows strong peak in the post-shock region, and the power gets enhanced from the latter part of the mid-sheath through the pre- and post-LE regions, and for strongest events the 


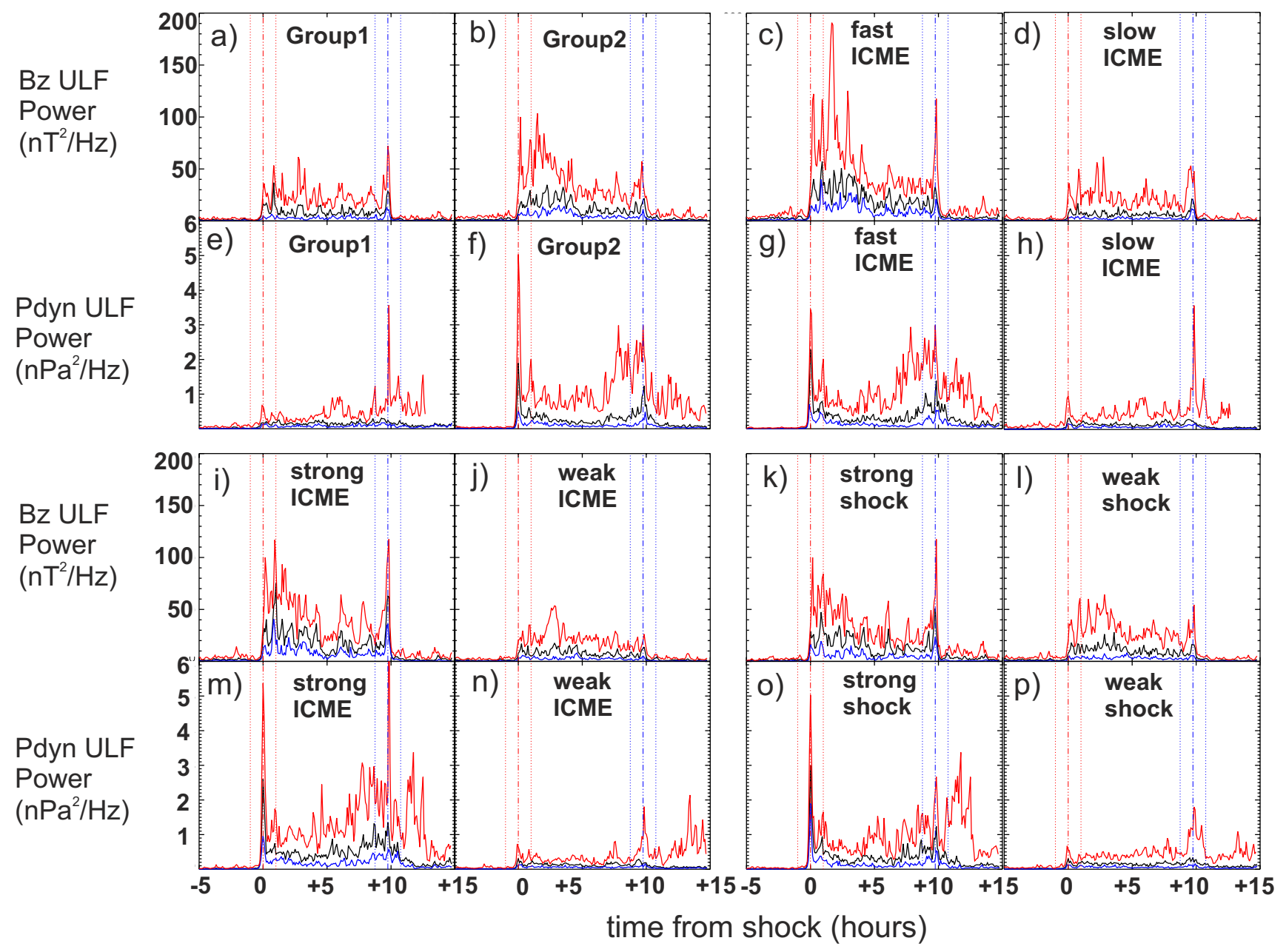

Fig. 4. $B_{Z}$ and $P_{\text {dyn }}$ ULF power separated into different categories (see Table 2).

power remains high also through the first hours of the ICME. The events associated with weak ICMEs have the strongest $P_{\text {dyn }}$ power in the post-LE region and within the ICME.

Interplanetary shocks have been shown to be preceded by regions of enhanced fluctuations in various frequency regimes (Greenstadt et al., 1982; Desai et al., 2011; Kajdič et al., 2012), i.e., by wave precursors or foreshocks. Our study concentrates on the sheath regions with much higher level of power, but some notions can be made about the before and pre-shock regions. In our data set, the main difference between events in the different categories was that for fast ICMEs, the upper quartile curve showed a clear increase in the power towards the shock, while for low-speed ICMEs the profile stayed relatively flat until the shock was reached. From Table 3 we also see that the average values of powers in the pre-shock region are higher than in the before-region. Differences are most pronounced for the $P_{\text {dyn }}$ ULF power. For example, the average $P_{\text {dyn }}$ power calculated from the upper quartile curve is 5.8 times higher in the pre-shock region than in the before region.

\section{Discussion}

We showed that $B_{Y}$ and $B_{Z}$ components exhibited roughly similar variations in the sheath region and in its surroundings, while $B_{X}$ had a considerably flatter profile and lower fluctuation power. The largest fluctuation power was in $B_{Z}$. We found that the IMF and $P_{\text {dyn }}$ ULF power have opposite profiles in the mid-sheath region (Fig. 3 and Table 3); the IMF ULF power (in particular in $B_{Z}$ and $B_{Y}$ ) is strongly enhanced during the first half of the mid-sheath, while the $P_{\text {dyn }}$ ULF power is more enhanced during the latter half of the mid-sheath, in particular for the strongest events. The mismatch in profiles could be explained by processes associated with the magnetic field evolution and $P_{\text {dyn }}$ piling in ICME sheaths as discussed below.

In ICME sheaths solar wind plasma piles in front of the ICME leading edge (Siscoe and Odstrcil, 2008), while the magnetic field magnitude has an irregular profile and tends to decrease towards the ICME leading edge (Kaymaz and Siscoe, 2006). The opposite behavior is found in the planetary 
magnetosheaths where plasma flows around the obstacle and magnetic field magnitude increases from the shock towards the object. The observations show that in the Earth's magnetosheath, the variations in the temperature anisotropy lead to different turbulent behavior behind the bow shock and close to the magnetopause (Lucek et al., 2005). We also note that it is likely that current sheets and other magnetic field irregularities close to the ICME leading edge have had time to interact and, for example, reconnect, while the magnetic field that has more recently passed through the shock in the front part of the sheath is less evolved.

Both IMF and $P_{\text {dyn }}$ ULF power showed strong peaks in the post-shock region and in the vicinity of the leading edge (in particular for $B_{Z}$ ). These peaks are likely related to the microscale physical processes taking place near these boundaries. In particular, several studies have reported intense wave activity and large amplitude magnetic field fluctuations within shock downstream regions (e.g., Kennel et al., 1982; Schwartz et al., 1996; Bamert et al., 2004; Kataoka et al., 2005; Kajdič et al., 2012). The neighborhood of the ICME leading edge is a much less studied region in terms of plasma wave activity and plasma instabilities.

The findings described above imply that magnetospheric activity associated with solar wind ULF fluctuations in sheath regions (e.g., changes in convection and plasma sheet dynamics, Pc5 waves) is expected to be strongest at the beginning of the sheath and around the ICME leading edge. In addition, the magnetospheric processes induced by IMF ULF fluctuations are generally expected before the effects from the $P_{\mathrm{dyn}}$ ULF fluctuations. It is a relevant as to question whether solar wind $P_{\mathrm{dyn}}$ and IMF ULF fluctuations drive different magnetospheric activity. Studies have shown that $P_{\text {dyn }}$ ULF fluctuations excite in particular compressional Pc5 waves in the magnetosphere and on the ground (e.g., Kessel, 2008; Liu et al., 2009), while IMF ULF fluctuations have been associated more strongly with toroidal Pc5 waves through enhanced Kelvin-Helmholtz instability on the magnetopause (Kessel et al., 2004). The details of these processes are beyond the scope of this paper. Weak or slow ICMEs, or those that are encountered centrally, have in general weak ULF power with low variability, and thus are not expected to stir the magnetosphere significantly.

The determination of geomagnetic consequences is further complicated because solar wind plasma and IMF properties change while while transmitting through the bow shock into the magnetosheath. Thus, it is expected that turbulent characteristics of the ICME sheath and embedded ULF fluctuations are also modified when the sheath interacts with the bow shock. The interaction begins already in the foreshock region, where a large variety of different plasma waves exist (e.g., Eastwood et al., 2005). It is also possible that part of the ULF fluctuations that eventually hit the magnetopause are generated by local sources in the foreshock and bow shock downstream regions (see e.g., Gutysnka et al., 2012; Hartinger et al., 2012, and references therein). The details of the ICME sheath-bow shock/magnetosheath interaction are a particularly interesting topic that we hope to investigate in the near future.

Sheath regions associated with strong or fast ICMEs had the strongest ULF power and rather similar power profiles (Fig. 4). These correlations might be expected as Gonzalez et al. (1998) showed that faster ICMEs tend to possess higher magnetic fields, and faster ICMEs should drive stronger shocks. However, the resemblance between the power levels and profiles for sheath regions of Group 2 and strong ICMEs is surprising. The magnetic field intensity should decrease with increasing impact parameter as ICMEs should have stronger fields in their core than close to their flanks. Nevertheless, such connections should be taken with caution because ICME properties vary considerably from event to event.

The high ULF power in sheath regions of Group 2 ICMEs could be related to stronger magnetic field line draping at the flanks of the ICME than at its nose (e.g., Gosling and McComas, 1987; Kaymaz and Siscoe, 2006). It is also possible that, due to geometrical constraints, the ICME shock nose is less likely to be quasi-parallel than the flanks. Thus, the higher turbulence associated to downstream regions of quasiparallel shocks could contribute to the stronger ULF power in Group 2 events.

The enhancement in the $B_{Z}$ ULF power in the post-shock region and in the first part of the mid-sheath was particularly strong for fast ICMEs. As mentioned above, faster ICMEs should have stronger shocks. The enhancement in power was indeed present for the strong shock category, but not as spectacularly. An additional property of the fast ICMEs is that their travel times from the Sun to the Earth are shorter, which may affect the level and nature of turbulence in their sheaths.

Due to the diversity of ICME-related in situ signatures it is often difficult to determine unambiguously the ICME boundaries (e.g., Richardson and Cane, 2010). As demonstrated in Fig. 3 the IMF ULF power drops sharply and abruptly when the spacecraft moves from the sheath into the ICME. The smoothness of the magnetic field is indeed one of the ICME identification criteria, but it is difficult to determine quantitatively. Thus, the IMF ULF power could possibly be used for identifying ICME front boundaries. It will be a future study to investigate in more detail how ULF activity behaves close to the ICME boundaries, and whether its variations could be used to distinguish between different ICME structures.

\section{Summary and conclusions}

In this paper we have studied how ULF fluctuations in IMF and in solar wind dynamic pressure $\left(P_{\mathrm{dyn}}\right)$ are distributed within CME-driven sheath regions using a superposed epoch analysis. The motivation for this study stemmed from the importance of sheath regions as drivers of magnetospheric 
activity and the lack of statistical studies on the large-scale sheath properties.

We found that the strongest peaks in the ULF power are concentrated just after the shock and in the vicinity of the ICME leading edge. The IMF ULF power was high in the leading part of the sheath, while the $P_{\text {dyn }}$ ULF power was most enhanced in the trailing part of the sheath. The mismatch in $P_{\text {dyn }}$ and IMF ULF power profiles within the sheath could be related to the effects from the piling of solar wind plasma in front of the ICME and general magnetic field evolution in sheath regions. Different IMF and $P_{\text {dyn }}$ power profiles may have significant consequences on the level and temporal evolution of the magnetospheric activity induced by sheath regions. The strongest ULF power was observed in sheath regions of strong or fast ICMEs, or ICMEs that were crossed at the intermediate distances from the center. We concluded that this is presumably attributed to stronger shocks driven by fast ICMEs and significant magnetic field line draping at the ICME flanks.

Deeper understanding of overall sheath properties and their geomagnetic consequences requires a more extensive analysis of magnetic field and solar wind fluctuations in different frequency ranges and their separate effects on the magnetosphere-ionosphere system. In addition, clues to how sheath properties evolve from the Sun to the Earth can be achieved by revisiting the Helios data and exploiting the observations from the European Space Agency's Solar Orbiter, scheduled for launch in 2017. These missions have their perihelion as close as $0.3 \mathrm{AU}$ from the Sun.

Acknowledgements. We thank N. Ness for the ACE MFI data, and D. J. McComas for the ACE SWE data. We thank L. Jian for the ACE ICME catalog. The Academy of Finland (project 1218152) and Väisälä Foundation are thanked for financial support. The work of Heli Hietala was funded by the UK Science and Technology Facilities Council (STFC).

Topical Editor C. Owen thanks the two anonymous referees for their help in evaluating this paper.

\section{References}

Baker, D. N., Pulkkinen, T. I., Li, K. X., and Kanekal, S. G.: Coronal mass ejections, magnetic clouds and relativistic magnetospheric electron events: ISTP, J. Geophys. Res., 103, 1727917291, 1998.

Bamert, K., Kallenbach, R., Ness, N. F., Smith, C. W., Terasawa, T., Hilchenbach, M., Wimmer-Schweingruber, R. F., and Klecker, B.: Hydromagnetic wave excitation upstream of an interplanetary traveling shock, Astrophys. J., 601, L99-L102, doi:10.1086/381962, 2004.

Blanco-Cano, X., Omidi, N., and Russell, C. T.: Macrostructure of collisionless bow shocks: 2. ULF waves in the foreshock and magnetosheath, J. Geophys. Res., 111, A10, doi:10.1029/2005JA011421, 2006.
Borovsky, J. E. and Funsten, H. O.: Role of solar wind turbulence in the coupling of the solar wind to the Earth's magnetosphere, J. Geophys. Res., 108, L20101, doi:10.1029/2002JA009601, 2002.

Desai, M. I., Dayeh, M. A., Smith, C. W., Lee, M. A., and Mason, G. M.: Ion acceleration near CME-Driven interplanetary shocks, in: 32nd International Cosmic Ray Conference, vol. 10, p. 53, 2011.

Eastwood, J. P., Lucek, E. A., Mazelle, C., Meziane, K., Narita, Y., Pickett, J., and Treumann, R. A.: The foreshock, Space Sci. Rev., 118, 41-94, 2005.

Golsing, J. T. and McComas, D. J.: Field line draping about fast coronal mass ejecta: A source of strong out-of-the-ecliptic interplanetary magnetic fields, Geophys. Res. Lett., 14, 355-358, 1987.

Gonzalez, W. D., de Gonzalez, A. L., Dal Lago, A., Tsurutani, B. T., Arballo, J. K., Lakhina, G. K., Buti, B., Ho, C. M., and Wu, S.-T.: Magnetic cloud field intensities and solar wind velocities, Sol. Phys., 25, 963-966, 1998.

Greenstadt, E. W., Scarf, F. L., Fredricks, R. W., Kennel, C. F., and Smith, E. J.: Plasma wave levels and IMF orientations preceding observations of interplanetary shocks by ISEE-3, J. Geophys. Res., 9, 668-671, doi:10.1029/GL009i006p00668, 1982.

Gutynska, O., Šimunek, J., Šafránková, J., Němeček, Z., and Přech, L.: Multipoint study of magnetosheath magnetic field fluctuations and their relation to the foreshock, J. Geophys. Res., 117, A4, doi:10.1029/2011JA017240, 2012.

Hartinger, M., Vassilis, A., Moldwin, M. B., Nishimura, Y., Turner, D. L., Glassmeier, K.-H., Kivelson, M. G., Matzka, J., and Stolle, C.: Observations of a Pc5 global (cavity/waveguide) mode outside the plasmasphere by THEMIS, J. Geophys. Res., 117, A6, doi:10.1029/2011JA017266, 2012.

Huttunen, K. E. J. and Koskinen, H. E. J.: Importance of post-shock streams and sheath region as drivers of intense magnetospheric storms and high-latitude activity, Ann. Geophys., 22, 1729-1738, doi:10.5194/angeo-22-1729-2004, 2004.

Huttunen, K. E. J., Koskinen, H. E. J., and Schwenn, R.: Variability of magnetospheric storms driven by different solar wind perturbations, J. Geophys. Res., 107, SMP 20-1-SMP 20-8, 2002.

Huttunen, K. E. J., Kilpua, S. P., Pulkkinen, A., Viljanen, A., and Tanskanen, E.: Solar wind drivers of large geomagnetically induced currents during the solar cycle 23, Space Weather, 6, S10002, doi:10.1029/2007SW000374, 2008.

Jian, L. K., Russell, C. T., Luhmann, J. G., and Skoug, R. M.: Properties of interplanetary coronal mass ejections at one AU during 1995-2004, Sol. Phys., 239, 393-436, 2006.

Kajdič, P., Blanco-Cano, X., Aguilar-Rodriguez, E, Russell, C. T., Jian, L. K., and Luhmann, J. G.: Waves upstream and downstream of interplanetary shocks driven by coronal mass ejections, J. Geophys. Res., 117, A6, doi:10.1029/2011JA017381, 2012.

Kataoka, R., Watari, S., Shimada, N., Shimazu, H., and Marubashi, K.: Downstream structures of interplanetary fast shocks associated with coronal mass ejections, Geophys. Res. Lett., 32, L12103, doi:10.1029/2005GL022777, 2005.

Kaymaz, Z. and Siscoe, G.: Field-Line Draping Around ICMES, Sol. Phys., 239, 437-448, 2006.

Kennel, C. F., Coroniti, F. V., Scarf, F. L., Smith, E. J., and Gurnett, D. A.: Nonlocal plasma turbulence associated with interplanetary shocks, J. Geophys. Res., 87, 17-34, doi:10.1029/JA087iA01p00017, 1982. 
Kepko, L., Spence, H. E., and Singer, H. J.: ULF waves in the solar wind as direct drivers of magnetospheric pulsations, Geophys. Res. Lett., 29, 39-1-39-4, doi:10.1029/2001GL014405, 2002.

Kessel, R. L.: Solar wind excitation of Pc5 fluctuations in the magnetosphere and on the ground, J. Geophys. Res., 113, A4, doi:10.1029/2007JA012255, 2008.

Kessel, R. L., Mann, I. R., Fung, S. F., Milling, D. K., and O'Connell, N.: Correlation of Pc5 wave power inside and outside themagnetosphere during high speed streams, Ann. Geophys., 22, 629-641, doi:10.5194/angeo-22-629-2004, 2004.

Kim, J., Lyons, L. R., Zou, S., Boudouridis, A., Lee, D.Y., Heinselman, C., and McCready, M.: Evidence that solar wind fluctuations substantially affect the strength of dayside ionospheric convection, J. Geophys. Res., 114, A11, doi:10.1029/2009JA014280, 2009.

Liu, W., Sarris, T. E., Li, X., Elkington, S. R., Ergun, R., Angelopoulos, V., Bonnell, J., and Glassmeier, K. H.: Electric and magnetic field observations of Pc4 and Pc5 pulsations in the inner magnetosphere: A statistical study, J. Geophys. Res., 114, A12, doi:10.1029/2009JA014243, 2009.

Lucek, E. A., Constantinescu, D., Goldstein, M. L., Pickett, J., Pinçon, J. L., Sahraoui, F., Treumann, R. A., and Walker, S. N.: The Magnetosheath, Space Sci. Rev., 118, 95-152, doi:10.1007/s11214-005-3825-2, 2005.

Luhmann, J. G., Russell, C. T., and Elphic, R. C.: Spatial distributions of magnetic field fluctuations in the dayside magnetosheath, J. Geophys. Res., 91, 1711-1715, doi:10.1029/JA091iA02p01711, 1986.

Lyons, L. R., Kim, H.-J., Xing, X., Zou, S., Lee, D.-Y., Heinselman, C., Nicolls, M. J., Angelopoulos, V., Larson, D., McFadden, J., Runov, A., and Fornacon, K.-H.: Evidence that solar wind fluctuations substantially affect global convection and substorm occurrence, J. Geophys. Res., 114, 11, doi:10.1029/2009JA014281, 2009.

Ogilvie, K., Chornay, D., Fritzenreiter, R., Hunsaker, F., Keller, J., Lobell, J., Miller, G., Scudder, J., Sittler, E. C., J., Torbert, R., Bodet, D., Needell, G., Lazarus, A., Steinberg, J., Tappan, J., Mavretic, A., and Gergin, E.: SWE, a comprehensive plasma instrument for the WIND spacecraft, Space Sci. Rev., 71, 55-77, doi:10.1007/BF00751326, 1995.
Richardson, I. G. and Cane, H. V.: Near-Earth interplanetary coronal mass ejections during solar cycle 23 (1996-2009): catalog and summary of properties, Sol. Phys., 264, 189-237, doi:10.1007/s11207-010-9568-6, 2010.

Sahraoui, F., Pincon, J.L., Belmont, G., Rezeau, L., CornilleauWehrlin, N., Robert, P., Mellul, L., Bosqued, J. M., Balogh, A., Canu, P., and Chanteur, G., ULF wave identification in the magnetosheath: The k-filtering technique applied to Cluster II data, J. Geophys. Res., 108, A9, doi:10.1029/2002JA009587, 2003.

Schwartz, S. J., Burgess, D., and Moses, J. J.: Low-frequency waves in the Earth's magnetosheath: present status, Ann. Geophys., 14, 1134-1150, doi:10.1007/s00585-996-1134-z, 1996.

Shevyrev, N. N., Zastenker, G. N., Eiges, P. E., and Richardson, J. D.: Low frequency waves observed by Interball-1 in foreshock and magnetosheath, Adv. Space Res., 37, 1516-1521, doi:10.1016/j.asr.2005.07.072, 2005.

Simms, L. E., Pilipenko, V. A., and Engebretson, M. J.: Determining the key drivers of magnetospheric Pc5 wave power, J. Geophys. Res., 115, A10, doi:10.1029/2009JA015025, 2010.

Siscoe, G. and Odstrcil, D.: Ways in which ICME sheaths differ from magnetosheaths, J. Geophys. Res., 113, A9, doi:10.1029/2008JA013142, 2008.

Smith, C., L'Heureux, J., Ness, N., Acuna, M., Burlaga, L., and Scheifele, J.: The ACE Magnetic Fields Experiment, Space Sci. Rev., 86, 613-632, doi:10.1023/A:1005092216668, 1998.

Tsurutani, B. T., Smith, E. J., Gonzalez, W. D., Tang, F., and Akasofu, S. I.: Origin of interplanetary southward magnetic fields responsible for major magnetic storms near solar maximum (19781979), J. Geophys. Res., 93, 8519-8531, 1998.

Yordanova, E., Vaivads, A., André, M., Buchert, S. C., and Vörös, Z.: Magnetosheath plasma turbulence and its spatiotemporal evolution as observed by the Cluster spacecraft, Phys. Rev. Lett., 100, 205003, doi:10.1103/PhysRevLett.100.205003, 2008. 